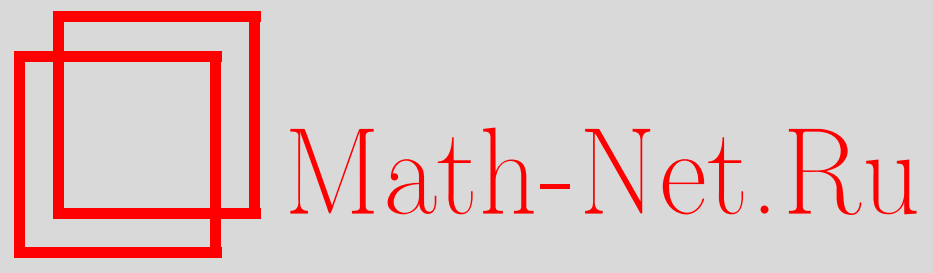

Н. В. Крамаренко, Обзор способов вывода критериев подобия в механике, Вестн. Сам. гос. техн. ун-та. Сер. Физ.-мат. науки, 2021, номер 1, 163 192

DOI: https://doi.org/10.14498/vsgtu1791

Использование Общероссийского математического портала MathNet.Ru подразумевает, что вы прочитали и согласны с пользовательским соглашением

http://www.mathnet.ru/rus/agreement

Параметры загрузки:

IP : 18.234 .197 .8

26 апреля 2023 г., 16:51:07

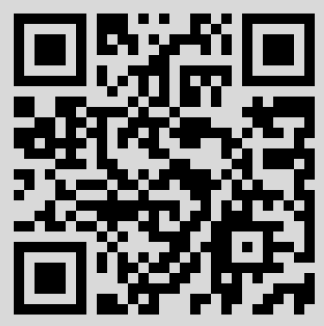


УДК 517.958:530.17

\section{Обзор способов вывода критериев подобия в механике}

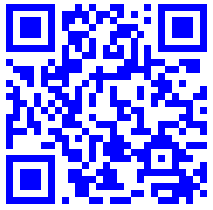

\section{(C) Н. В. Крамаренко}

Новосибирский государственный технический университет, Россия, 630073, Новосибирск, пр-т Карла Маркса, 20.

\section{Аннотация}

Дается обзор различных способов получения критериев подобия, приводится их классификация, которая включает пять способов их вывода из дифференциальных уравнений и семь способов - из анализа размерностей. Все способы сравниваются на одной задаче механики о вынужденных колебаниях груза, приводящей к четырем числам подобия. Такой подход помогает сравнить трудозатраты, необходимые для вывода чисел подобия различными способами. По каждому способу дается перечень литературы, где он упоминается, и краткое описание задач, которые там решаются. В конце приводится сводная таблица, показывающая, какие способы рассмотрены в упоминаемых работах. Из таблицы видна сравнительная популярность способов.

Ключевые слова: теория подобия, методы подобия, критерии подобия, числа подобия, индикаторы подобия, анализ размерностей.

Получение: 12 июня 2020 г. / Исправление: 2 марта 2021 г. /

Принятие: 10 марта 2021 г. / Публикация онлайн: 29 марта 2021 г.

\section{Введение}

О преподавании. Теория подобия преподается во многих вузах. О желательности преподавания теории подобия для студентов технических специальностей говорится уже давно. Например, в 1979 году на Всесоюзном совещании-семинаре заведующих кафедрами механики вузов страны Л. Г. Лойцянский в своем докладе [26] отмечал «важность введения в преподаваемые курсы теоретической механики хотя бы элементарных сведений о подобии и размерности». В 1998 году такую же мысль высказывал И. Ш. Коган [17]: «Основы теории подобия следует излагать перед началом изучения технических дисциплин, с тем чтобы применять полученные знания при изучении каждой из них».

\section{Обзор}

( () (7) Контент публикуется на условиях лицензии Creative Commons Attribution 4.0 International (https://creativecommons.org/licenses/by/4.0/deed.ru)

\section{Образец для цитирования}

Крамаренко Н. В. Обзор способов вывода критериев подобия в механике // Вестн. Сам. гос. техн. ун-та. Сер. Физ.ммат. науки, 2021. Т. 25, № 1. С. 163-192. https://doi.org/10.14498/vsgtu1791.

\section{Сведения об авторе}

Николай Владимирович Крамаренко (D) https://orcid.org/0000-0002-6301-1255 кандидат технических наук, доцент; каф. прочности летательных аппаратов; e-mail: kramnv@rambler.ru ; e-mail:n.kramarenko@corp.nstu.ru 
О терминах. В литературе используются термины «методы подобия» и «теория подобия». Какой более правильный? С общефизической точки зрения теорией можно называть такой раздел физики, где изучаются те или иные реальные физические процессы, происходящие с веществом или полем. Поэтому с этих позиций правильнее говорить «методы подобия» в той или иной физической теории, по аналогии с названиями других методов, например, численные методы, вариационные методы, методы оптимизации. С математической точки зрения все методы решения физических задач имеют свою теорию, и с этих позиций естественно говорить «теория подобия». В данной работе будем употреблять оба термина.

Кроме того, в литературе используется термин «теория подобия и анализ размерностей», т. е. теория подобия противопоставляется анализу размерностей. Такое противопоставление может сформировать неправильное понимание предмета. Для целостного восприятия методов подобия будем вместо термина «теория подобия» использовать термин «анализ уравнений». Таким образом, теория подобия делится на два направления: анализ уравнений и анализ размерностей. Такая терминология не нова, она встречается, например, в работах М. В. Кирпичева [14, с. 90], С. С. Кутателадзе [23, введение]. Добавим, что в [23] термины «теория» и «методы» подобия заменены единым термином «анализ» подобия.

Еще одна неоднозначность возникает при употреблении терминов критерий подобия и число подобия. Согласно сборнику рекомендуемых терминов по теории подобия [32], эти термины равнозначны, и на английском языке они обозначаются единым выражением similarity criterion. Для того чтобы отличать входные параметры задачи от выходных, в [32] вводятся понятия определяющего и определяемого критериев подобия. Под определяющим (англ. independent similarity criterion) понимается критерий подобия, содержащий независимую переменную, под определяемым (англ. dependent similarity criterion) - критерий подобия, содержащий зависимую переменную (искомую величину). Для того чтобы выделить эти различия, например, Л. А. Шаповалов [44] вводит подчеркивание в критериальном уравнении:

$$
\pi_{1}=f\left(\underline{\left.\pi_{2}, \pi_{3}, \ldots\right)} .\right.
$$

Здесь подчеркнуты определяющие критерии, слева - определяемый критерий подобия. В противоположность сборнику [32] Л. Г. Лойцянский [24-26] для разграничения понятий называет критериями только определяющие критерии подобия, а под числами подобия понимает все критерии - и определяющие, и определяемые: «критериев подобия меньше, чем чисел подобия». В качестве обоснования такой позиции Л. Г. Лойцянский приводит рассуждение о том, что если равны критерии, то подобие двух сравниваемых явлений («модели» и «натуры») будет обеспечено независимо от остальных чисел подобия.

Об обозначениях. Традиционно безразмерные числа и критерии подобия обозначаются прописной или строчной греческой буквой «пи». Такое обозначение возникло, вероятно, от первой буквы греческого слова тарацєтро (параметр, аргумент) ${ }^{1}$.

О получении критериев подобия. В классическом представлении числа подобия, или инварианты, можно получить двумя путями: либо из диффе-

\footnotetext{
${ }^{1}$ По материалам сайта Wikipedia.
} 
ренциальных уравнений, описывающих процесс или явление, либо из анализа размерностей параметров задачи.

\section{1. Способы вывода критериев подобия из дифференциальных уравнений}

Рассмотрим следующие пять способов вывода чисел подобия из дифференциальных уравнений, которые для краткости обозначим через Д1-Д5:

Д1. Способ нормализации уравнения, или приведения уравнения к безразмерному виду;

Д2. Способ упрощенной нормализации уравнения;

Д3. Способ деления уравнения на одно из слагаемых;

Д4. Способ сравнения двух уравнений через индикаторы подобия;

Д5. Способ сравнения двух сил через индикаторы подобия.

Общие положения по выводу чисел подобия будем иллюстрировать примерами для задачи о вынужденных колебаниях груза, которые описываются уравнением

$$
m \frac{d^{2} x}{d t^{2}}+k \frac{d x}{d t}+c x=P \sin \omega t
$$

где $m$ - масса груза (материальной точки); $k$ - коэффициент демпфирования; $c$-жесткость пружины; $P, \omega$ - амплитуда и круговая частота возмущающей силы. В этом уравнении четыре слагаемых, и каждое слагаемое есть сила: первое - сила инерции $F_{m}$, второе - сила сопротивления $F_{k}$, третьесила упругости $F_{c}$, справа стоит внешняя возмущающая сила $F_{\omega}$, например, от вращающегося дебаланса:

$$
F_{m}=m \frac{d^{2} x}{d t^{2}}, \quad F_{k}=k \frac{d x}{d t}, \quad F_{c}=c x, \quad F_{\omega}=P \sin \omega t
$$

Д1. Способ нормализации уравнения, или приведения уравнения к безразмерному виду. Выберем характерные значения для аргумента $t$, функции $x$ и параметров

$$
t_{*}, x_{*}, m_{*}, k_{*}, c_{*}, P_{*}, \omega_{*} .
$$

На первом этапе рассуждений эти величины остаются произвольными. Определим соответствующие безразмерные величины:

$$
\bar{t}=\frac{t}{t_{*}}, \quad \bar{x}=\frac{x}{x_{*}}, \quad \bar{m}=\frac{m}{m_{*}}, \quad \bar{k}=\frac{k}{k_{*}}, \quad \bar{c}=\frac{c}{c_{*}}, \quad \bar{P}=\frac{P}{P_{*}}, \quad \bar{\omega}=\frac{\omega}{\omega_{*}} .
$$

Тогда размерные величины и размерные производные примут следующий вид:

$$
\begin{gathered}
t=\bar{t} t_{*}, \quad x=\bar{x} x_{*}, \quad m=\bar{m} m_{*}, \quad k=\bar{k} k_{*}, \quad c=\bar{c} c_{*}, \quad P=\bar{P} P_{*}, \quad \omega=\bar{\omega} \omega_{*} ; \\
\frac{d x}{d t}=\left(\frac{x_{*}}{t_{*}}\right) \frac{d \bar{x}}{d \bar{t}}, \quad \frac{d^{2} x}{d t^{2}}=\frac{d}{d t}\left(\frac{d x}{d t}\right)=\left(\frac{x_{*}}{t_{*}^{2}}\right) \frac{d^{2} \bar{x}}{d \bar{t}^{2}} .
\end{gathered}
$$


Подставим их в уравнение (1):

$$
\left(m_{*} \frac{x_{*}}{t_{*}^{2}}\right) \bar{m} \frac{d^{2} \bar{x}}{d \bar{t}^{2}}+\left(k_{*} \frac{x_{*}}{t_{*}}\right) \bar{k} \frac{d \bar{x}}{d \bar{t}}+\left(c_{*} x_{*}\right) \overline{c x}=P_{*} \bar{P} \sin \left(\omega_{*} t_{*} \overline{\omega t}\right) .
$$

Поделим все слагаемые на первую скобку:

$$
\bar{m} \frac{d^{2} \bar{x}}{d \bar{t}^{2}}+\left(\frac{k_{*} t_{*}}{m_{*}}\right) \bar{k} \frac{d \bar{x}}{d \bar{t}}+\left(\frac{c_{*} t_{*}^{2}}{m_{*}}\right) \overline{c x}=\left(\frac{P_{*} t_{*}^{2}}{m_{*} x_{*}}\right) \bar{P} \sin \left(\left(\omega_{*} t_{*}\right) \overline{\omega t}\right) .
$$

Далее проведем следующие логические рассуждения:

1) здесь первое слагаемое есть величина безразмерная, следовательно, все остальные слагаемые тоже должны быть безразмерными по правилу одинаковых размерностей Фурье;

2) в каждом слагаемом скобка умножается на безразмерную величину, следовательно, каждая скобка тоже должна быть величиной безразмерной.

Обозначим выражения в скобках через безразмерные параметры $\pi$ и получим безразмерное уравнение

$$
\bar{m} \frac{d^{2} \bar{x}}{d \bar{t}^{2}}+\pi_{1} \bar{k} \frac{d \bar{x}}{d \bar{t}}+\pi_{2} \overline{c x}=\pi_{3} \bar{P} \sin \left(\pi_{4} \overline{\omega t}\right) .
$$

Здесь первые три безразмерных параметра $\pi$ имеют физический смысл отношения соответствующих сил, а четвертый - угла поворота дебаланса:

$$
\pi_{1}=\frac{F_{k}}{F_{m}}=\frac{k_{*} t_{*}}{m_{*}}, \quad \pi_{2}=\frac{F_{c}}{F_{m}}=\frac{c_{*} t_{*}^{2}}{m_{*}}, \quad \pi_{3}=\frac{F_{\omega}}{F_{m}}=\frac{P_{*} t_{*}^{2}}{m_{*} x_{*}}, \quad \pi_{4}=\omega_{*} t_{*} .
$$

На втором этапе рассуждений характерным размерным величинам (3) следует задать конкретные значения. Если эти значения будут близки к максимально возможным для этой задачи, то безразмерное уравнение (4) нормализуется, т. е. каждое слагаемое уравнения будет по модулю меньше единицы или равно ей. Зададим следующие значения:

$$
\begin{aligned}
& t_{*}=t_{p}, \quad x_{*}=x_{s}=P / c, \quad m_{*}=m, \\
& k_{*}=k, \quad c_{*}=c, \quad P_{*}=P, \quad \omega_{*}=\omega_{0}=\sqrt{c / m} .
\end{aligned}
$$

Здесь $t_{p}$ - время периода колебаний; $x_{s}$ - статическое отклонение груза под действием постоянной силы $P ; m, k, c, P$-соответствующие параметры задачи; $\omega_{0}$ - частота собственных колебаний без демпфирования. Тогда безразмерное отклонение $\bar{x}$ и безразмерное время $\bar{t}$ будут измеряться в долях от статического отклонения $x_{s}$ и от периода колебаний $t_{p}$ соответственно:

$$
\bar{x}=\frac{x}{x_{*}}=\frac{x}{x_{s}}, \quad \bar{t}=\frac{t}{t_{*}}=\frac{t}{t_{p}},
$$

а безразмерные масса, коэффициент демпфирования, жесткость пружины и внешняя сила будут равны единице:

$$
\bar{m}=\frac{m}{m_{*}}=\frac{m}{m}=1, \quad \bar{k}=\frac{k}{k_{*}}=\frac{k}{k}=1, \quad \bar{c}=\frac{c}{c_{*}}=\frac{c}{c}=1, \quad \bar{P}=\frac{P}{P_{*}}=\frac{P}{P}=1 .
$$


В результате уравнение (4) и коэффициенты $\pi$ (5) примут вид

$$
\begin{gathered}
\frac{d^{2} \bar{x}}{d \bar{t}^{2}}+\pi_{1} \frac{d \bar{x}}{d \bar{t}}+\pi_{2} \bar{x}=\pi_{3} \sin \left(\pi_{4} \overline{\omega t}\right) ; \\
\pi_{1}=\frac{k t_{p}}{m}, \quad \pi_{2}=\frac{c t_{p}^{2}}{m}, \quad \pi_{3}=\frac{P t_{p}^{2}}{m x_{s}}, \quad \pi_{4}=\omega_{0} t_{p} .
\end{gathered}
$$

В теории подобия безразмерные коэффициенты $\pi$ получили название чисел (критериев) подобия. Число $\pi_{4}$ называется числом гомохронности, число $\pi_{3}$ называется числом подобия Ньютона (отношение внешней силы к силе инерции). В дальнейшем у времени $t_{p}$, смещения $x_{s}$ и частоты $\omega_{0}$ индексы для краткости не будем писать, но подразумеваем, что это конкретные характерные значения (6).

Уравнение в безразмерном виде имеет три преимущества по сравнению с размерным уравнением:

1) из условия безразмерности чисел подобия $\pi$ можно без решения дифференциального уравнения (7) провести сравнительный анализ параметров задачи, т. е. определить, во сколько раз изменится какой-либо параметр, если изменятся другие параметры;

2) приведение уравнения к безразмерному виду позволяет в общем случае сократить количество параметров, от которых зависит искомая функция, а это облегчает как проведение экспериментов, так и дальнейший анализ задачи;

3) безразмерная форма уравнений позволяет проводить аналоговое моделирование процесса в другой физической среде, например, изучать колебания сложной механической системы на электрической схеме. Такие исследования проводились на аналоговых ЭВМ до появления цифровых компьютеров с развитыми численными методами.

В результате получили, что натура и модель описываются одинаковыми безразмерными уравнениями (7), отличия только в характерных параметрах (6) и безразмерных комплексах $\pi_{1}, \pi_{2}, \pi_{3}, \pi_{4}$. Для подобия натуры и модели требуется, чтобы их $\pi$-коэффициенты совпадали:

$$
\pi_{1}=\frac{k t}{m}=\text { idem, } \pi_{2}=\frac{c t^{2}}{m}=\text { idem, } \pi_{3}=\frac{P t^{2}}{m x}=\text { idem, } \pi_{4}=\omega t=\text { idem },
$$

где idem в буквальном переводе с латинского означает «одно и то же».

Способ Д1 описан под разными названиями у многих авторов и применялся для различных задач.

К. С. Басниев и другие [6] рассматривают задачу о приведении уравнений к безразмерному виду на примере системы уравнений движения однородной вязкой несжимаемой жидкости. Из уравнения движения и граничных условий получают числа подобия Фруда, Рейнольдса, Эйлера, Струхаля. Для выяснения физического смысла чисел подобия авторы рассматривают в жидкости параллелепипед, на который действуют сила тяжести, сила локальной инерции, сила конвективной инерции и сила трения. Далее показывают, что числа подобия есть отношения этих сил. 
Г. Биркгоф [7] со ссылкой на Руарка [54] называет этот способ инспекционным анализом и отмечает, что он основан на старой идее, которая была предложена Эренфест-Афанасьевой [51,52]. Инспекционный анализ - это нормализация дифференциальных уравнений и оценка малости отдельных слагаемых. Г. Биркгоф показывает применение этого способа на уравнениях Навье-Стокса для несжимаемой вязкой жидкости и получает числа Рейнольдса, Фруда, кавитации. На основе сравнения их величин делает следующие выводы о необходимых условиях приближенного моделирования:

- если влияние силы тяжести, сжимаемости и кавитации незначительно, то модель должна иметь то же самое число Рейнольдса;

- если не имеют значения сжимаемость, кавитация и вязкость, то моделировать надо по числу Фруда;

- если можно пренебречь сжимаемостью и вязкостью, но надо учитывать гравитационные и кавитационные эффекты, то следует сохранять неизменными число Фруда и число кавитации.

В. А. Винников и Г. Г. Каркашадзе [10] числа подобия Фруда, Рейнольдса, Эйлера, а также число Струхаля для вязких несжимаемых жидкостей получают из уравнения Навье-Стокса в векторной форме, которое приводят к безразмерному виду. Такую запись называют уравнением движения НавьеСтокса в критериальной форме.

А. А. Гухман [11] безразмерные уравнения называет уравнениями в относительной форме и получает числа подобия в трех задачах:

1) в задаче о температурном поле в твердом теле из уравнения теплопроводности выводит число Фурье, из уравнения для граничных условий выводит число Био;

2) в задаче о движении сплошной среды из уравнений сплошности и движения выводит числа Рейнольдса, Фруда, Әйлера;

3) в задаче о переносе тепла в движущейся среде выводит числа Пекле, Прандтля, Нуссельта, Маха.

В работах $[20,21]$ способом Д1 решаются две прикладные задачи. В работе [20] рассматривается задача о моделировании с помощью теории подобия свободных движений твердого тела в гравитационном поле, в работе [21] решается задача о моделировании жесткости балки.

А. А. Кудинов [22] на примере одномерного движения несжимаемой жидкости показывает приведение к безразмерному виду дифференциального уравнения Навье-Стокса и уравнения неразрывности с граничными условиями. Отмечает, что если исходная система уравнений содержала 11 переменных, то система уравнений в безразмерном виде содержит всего 8 обобщенных переменных. Сокращение числа переменных упрощает исследование.

C. С. Кутателадзе [23] рассматривает сложные задачи гидромеханики с учетом влияния температуры и массопереноса и при этом использует прием сведения дифференциальных уравнений к безразмерному виду.

Л. Г. Лойцянский $[24,25]$ при рассмотрении различных задач гидромеханики также использует этот прием.

В. С. Швыдкий и другие [28] получают условия, при которых достигается динамическое подобие двух течений, путем записи уравнений движения жидкости в безразмерной форме и приравнивания числовых коэффициентов в обеих системах. 
Б. Т. Породнов [34] рассматривает изотермическое уравнение движения для невязких одноатомных газов и уравнение непрерывности. После приведения этих уравнений к безразмерному виду получает числа динамического подобия потоков Струхаля, Майевского, Рейнольдса, Фруда.

О. Я. Романов и В. В. Ходосов [35] кратко описывают этот способ, но примеров не приводят.

Дж. Серрин [39] кратко обсуждает вопросы динамического подобия. Замечает, что понятие динамического подобия принадлежит Стоксу (G. Stokes). В его работе [55] 1850 года о движении маятника в тормозящей жидкой среде не только впервые было сформулировано понятие динамического подобия, но и впервые фигурировала комбинация параметров течения, носящая сейчас название числа Рейнольдса.

С. С. Силин [40] использует способ безразмерных уравнений для задач резания материалов. Приводит формулу для безразмерной величины - числа пластичности В, характеризующего степень пластических деформаций металла снимаемого припуска и поверхностного слоя обрабатываемой детали. В эту формулу входят три безразмерных комплекса (числа подобия):

- число Пекле Ре, характеризующее степень влияния режимных условий процесса по сравнению с влиянием теплофизических свойств обрабатываемого материала;

- число F, отражающее влияние геометрии инструмента и отношения теплопроводностей инструментального и обрабатываемого материалов;

- число D, характеризующее геометрию сечения среза.

Этот способ также описывает В. А. Соколов [41].

Л. А. Шаповалов [44] со ссылкой на работу С. Дж. Клайна [16] отмечает, что под нормализацией физических уравнений и соответствующих им начальных и краевых условий подразумевается процедура, состоящая из двух операций:

1) уравнения приводятся к безразмерному виду;

2) все безразмерные параметры ограничиваются единицей.

Для успешной нормализации исходных размерных уравнений необходимо в качестве характерных размерных параметров выбрать их максимальные значения. Тогда соответствующие им безразмерные величины будут принимать значения в диапазоне от нуля до единицы. Нормализация помогает отбросить малые члены уравнений и затем проводить приближенное моделирование без соблюдения некоторых исходных критериев подобия.

Дальнейшим развитием способа нормализации уравнений является переход к естественным координатам, т. е. такое преобразование безразмерных аргументов, функций и параметров, чтобы в безразмерном уравнении не осталось параметров, и тогда безразмерная функция будет зависеть только от безразмерных аргументов без параметров. Такой способ преобразования переменных в [44] рассматривается на примере моделирования собственных поперечных колебаний балки постоянного поперечного сечения.

Л. А. Эпштейн [46] использует способ приведения уравнений к безразмерному виду в различных задачах гидромеханики.

Д2. Способ упрощенной нормализации уравнения. Суть способа состоит в том, что уравнение приводится к безразмерному виду путем умножения на алгебраический комплекс с обратной размерностью и затем у каж- 
дого одночлена отбрасываются знаки дифференциалов. В нашей задаче о вынужденных колебаниях груза в исходном уравнении (1) каждое слагаемое имеет размерность силы. Умножив его на величину с обратной размерностью

$$
m \frac{d^{2} x}{d t^{2}}+k \frac{d x}{d t}+c x=P \sin (\omega t) \quad \mid \times \frac{t^{2}}{m x},
$$

получим

$$
\frac{t^{2}}{x} \frac{d^{2} x}{d t^{2}}+\frac{t^{2} k}{m x} \frac{d x}{d t}+\frac{t^{2} c}{m}=\frac{P t^{2}}{m x} \sin (\omega t) .
$$

Отбросив знаки дифференциалов, в результате получаем в скобках те же числа подобия (8):

$$
\begin{aligned}
& \frac{t^{2}}{x} \frac{x}{t^{2}}+\frac{t^{2} k}{m x} \frac{x}{t}+\frac{t^{2} c}{m}=\frac{P t^{2}}{m x} \sin (\omega t) ; \\
& 1+\left(\frac{k t}{m}\right)+\left(\frac{c t^{2}}{m}\right)=\left(\frac{P t^{2}}{m x}\right) \sin (\omega t) .
\end{aligned}
$$

М. В. Кирпичев [14] этот способ называет методом относителъных единии. Для определения подобия он использует два термина: константы подобия (индикаторы) и инварианты (критерии) в зависимости от того, какое определение оказывается более удобным в смысле простоты изложения. Применяя способ упрощенной нормализации, из уравнения динамики точки М. В. Кирпичев выводит число Ньютона, из уравнения Навье-Стокса находит числа Фруда, Эйлера, Рейнольдса, а из них - числа Лагранжа, Галилея, Кирхгофа.

Р. Х. Санников [36] отмечает, что при получении чисел подобия по известным уравнениям исследуемых процессов возможны два случая.

Случай 1. Все члены уравнения - однородные функции параметров и их производных. При этом все члены уравнения имеют общий множитель, который может быть вынесен за скобки. В этом случае все члены уравнения делятся на какой-либо из них, и посредством элементарных преобразований получают числа подобия. В нашей задаче о колебаниях груза такой случай возникает в случае собственных колебаний, когда в правой части стоит ноль.

Случай 2. Часть членов уравнения - неоднородные функции параметров, не допускающие выноса за знак функции общего множителя. В этом случае числа подобия могут быть получены методом «интегральных аналогов». При этом используется то свойство подобных процессов, должны быть равны аргументы неоднородных функций. Далее в [36] описывается порядок действий из 8 пунктов. Такой алгоритм соответствует нашему способу Д2 упрощенной нормализации уравнения.

Д3. Способ деления уравнения на одно из слагаемых. Суть способа состоит в том, что исходное дифференциальное уравнение записывается через размерности, а затем оно делится на одно из слагаемых. Рассмотрим нашу задачу о вынужденных колебаниях груза. Представим уравнение движения (1) через размерности, входящие в него:

$$
\left[m \frac{d^{2} x}{d t^{2}}\right]+\left[k \frac{d x}{d t}\right]+[c x]=[P \sin (\omega t)], \quad\left[m \frac{x}{t^{2}}\right]+\left[k \frac{x}{t}\right]+[c x]=[P \sin (\omega t)] .
$$


Разделим все члены уравнения на первое слагаемое:

$$
1+\left[\frac{k t}{m}\right]+\left[\frac{c t^{2}}{m}\right]=\left[\frac{P t^{2}}{m x}\right][\sin (\omega t)] .
$$

В скобках последнего равенства находим те же числа подобия (8).

П. М. Алабужев и др. [1] показывают применение этого способа на примере вынужденных колебаний груза.

В. А. Веников [9] называет этот способ методом интегральных аналогов и показывает его применение на том же примере вынужденных колебаний груза.

М. В. Кирпичев [14] также описывает этот способ, но примеров не приводит.

Д4. Способ сравнения двух уравнений через индикаторы подобия. В нашей задаче о вынужденных колебаниях груза натурный объект оставим без номера, а модельный обозначим номером 1. Запишем для них уравнения процесса - для натуры это будет уравнение (1), для модели - похожее уравнение:

$$
m_{1} \frac{d^{2} x_{1}}{d t_{1}^{2}}+k_{1} \frac{d x_{1}}{d t_{1}}+c_{1} x_{1}=P_{1} \sin \left(\omega_{1} t_{1}\right)
$$

Введем коэффициенты подобия:

$$
t_{c}=\frac{t_{1}}{t}, \quad x_{c}=\frac{x_{1}}{x}, m_{c}=\frac{m_{1}}{m}, \quad k_{c}=\frac{k_{1}}{k}, \quad c_{c}=\frac{c_{1}}{c}, P_{c}=\frac{P_{1}}{P}, \omega_{c}=\frac{\omega_{1}}{\omega} .
$$

Определим параметры модели и ее производные через параметры натуры:

$$
\begin{gathered}
t_{1}=t_{c} t, \quad x_{1}=x_{c} x, \quad m_{1}=m_{c} m \\
k_{1}=k_{c} k, \quad c_{1}=c_{c} c, \quad P_{1}=P_{c} P, \quad \omega_{1}=\omega_{c} \omega ; \\
\frac{d x_{1}}{d t_{1}}=\frac{x_{c}}{t_{c}} \frac{d x}{d t}, \quad \frac{d^{2} x_{1}}{d t_{1}^{2}}=\frac{x_{c}}{t_{c}^{2}} \frac{d^{2} x}{d t^{2}} .
\end{gathered}
$$

Подставим эти значения в уравнение модели (9) и сгруппируем коэффициенты подобия:

$$
\left(\frac{m_{c} x_{c}}{t_{c}^{2}}\right) m \frac{d^{2} x}{d t^{2}}+\left(\frac{k_{c} x_{c}}{t_{c}}\right) k \frac{d x}{d t}+\left(c_{c} x_{c}\right) c x=\left(P_{c}\right) P \sin \left(\left(\omega_{c} t_{c}\right) \omega t\right)
$$

Для того чтобы модель была подобна натуре, необходимо, чтобы уравнение модели (11) совпадало с уравнением натуры (1). Это означает, что четыре комплексных коэффициента должны равняться между собой, а коэффициент под синусом должен равняться единице:

$$
\frac{m_{c} x_{c}}{t_{c}^{2}}=\frac{k_{c} x_{c}}{t_{c}}=c_{c} x_{c}=P_{c}, \quad \omega_{c} t_{c}=1
$$


Первые три равенства поделим на один из членов, например, на первый:

$$
1=\frac{k_{c} t_{c}}{m_{c}}=\frac{c_{c} t_{c}^{2}}{m_{c}}=\frac{P_{c} t_{c}^{2}}{m_{c} x_{c}} .
$$

В результате получим четыре уравнения для индикаторов подобия:

$$
\lambda_{1}=\frac{k_{c} t_{c}}{m_{c}}=1, \quad \lambda_{2}=\frac{c_{c} t_{c}^{2}}{m_{c}}=1, \quad \lambda_{3}=\frac{P_{c} t_{c}^{2}}{m_{c} x_{c}}=1, \quad \lambda_{4}=\omega_{c} t_{c}=1 .
$$

Таким образом, если уравнение модели (11) совпадает с уравнением натуры (1), то все индикаторы подобия (12) равны единице, и модель будет подобна натуре. Подставим значения коэффициентов подобия в индикаторы подобия:

$$
\begin{gathered}
\lambda_{1}=\frac{k_{c} t_{c}}{m_{c}}=1, \quad \frac{k_{1}}{k} \frac{t_{1}}{t} \frac{m}{m_{1}}=1, \quad \frac{k_{1} t_{1}}{m_{1}}=\frac{k t}{m} \\
\lambda_{2}=\frac{c_{c} t_{c}^{2}}{m_{c}}=1, \quad \frac{c_{1}}{c} \frac{t_{1}^{2}}{t^{2}} \frac{m}{m_{1}}=1, \quad \frac{c_{1} t_{1}^{2}}{m_{1}}=\frac{c t^{2}}{m} \\
\lambda_{3}=\frac{P_{c} t_{c}^{2}}{m_{c} x_{c}}=1, \quad \frac{P_{1}}{P} \frac{t_{1}^{2}}{t^{2}} \frac{m}{m_{1}} \frac{x}{x_{1}}=1, \quad \frac{P_{1} t_{1}^{2}}{m_{1} x_{1}}=\frac{P t^{2}}{m x} \\
\lambda_{4}=\omega_{c} t_{c}=1, \quad \frac{\omega_{1}}{\omega} \frac{t_{1}}{t}=1, \quad \omega_{1} t_{1}=\omega t
\end{gathered}
$$

Отсюда следуют те же числа подобия (8).

Таким образом, видим, что числа подобия $\pi$ получаются из индикаторов подобия $\lambda$ формальной заменой коэффициентов подобия на соответствующие параметры, т.е. путем отбрасывания индексов $c$.

В. Л. Кирпичев [13] для иллюстрации способа сравнения двух уравнений рассматривает подобие механических движений двух точек и через коэффициенты подобия находит число подобия Ньютона.

М. В. Кирпичев [14] способ Д4 называет методом констант подобия и иллюстрирует его на двух примерах. В первом примере рассматривает самый простой случай безвихревого течения вязкой жидкости между двумя бесконечными параллельными стенками. Уравнение Навье-Стокса и уравнение сплошности в этом случае принимают очень простой вид. Во втором примере рассматривает общий случай ламинарного течения жидкости в канале, имеющем переменные сечения и направления. Уравнениями связи для этого случая будут уравнение сплошности и уравнение движения потока НавьеСтокса. Здесь получается число гомохронности, числа Фруда, Эйлера, Рейнольдса, Лагранжа, числа Галилея, Кирхгофа. Через индикаторы подобия из уравнения динамики точки также получено число Ньютона.

С. Д. Корнеев [18] способом Д4 из уравнения динамики точки выводит динамический закон подобия Ньютона.

А. А. Кудинов [22] при выводе чисел подобия использует способ двух уравнений - для натуры и модели, получает индикатор, а затем число подобия Ньютона.

В. С. Швыдкий и др. [28] рассматривают уравнения для двух подобных потоков, из которых устанавливают коэффициенты подобия, а затем индикаторы и числа подобия Ньютона, Фруда, Рейнольдса, Эйлера. 
О. Я. Романов и В. В. Ходосов [35], а также Р. Х. Санников [36] кратко описывают этот способ, но примеров не приводят.

О. С. Сергель [38] получает безразмерные числа подобия из уравнений Навье-Стокса одномерного течения для натуры и модели. Уравнение движения, описывающее модельное течение, записывает в параметрах натурного и, сравнивая их, получает индикаторы подобия, а из них - число Струхаля или временной однородности (гомохронности), число Фруда, число Эйлера, число Рейнольдса.

Л. А. Шаповалов [44] называет способ сравнения двух уравнений через индикаторы подобия методом масштабных преобразований. Применяя этот способ к задаче изгиба консольной балки сосредоточенной силой и моментом, показывает, что условия механического подобия не зависят от операторов дифференцирования в физических уравнениях. Для этого он сначала рассматривает выражения для нормального и касательного напряжений, осевой деформации и поперечного перемещения в алгебраической форме, а затем дифференциальные уравнения изгиба. Сравнивая результаты, показывает, что масштабные преобразования краевой задачи как для дифференциальных уравнений, так и для интеграла этих уравнений в алгебраической форме дают одинаковый результат.

Д5. Способ сравнения двух сил через индикаторы подобия. Суть способа состоит в том, что вместо всего уравнения рассматриваются отдельные его слагаемые. Такой подход используется при рассмотрении задач, которые описываются несколькими уравнениями, и применение для них предыдущего способа Д4 приводит к громоздким выкладкам. Кроме того, этот способ выявляет физический смысл чисел подобия. При таком подходе базовым критерием подобия выступает число Ньютона, в котором произвольная сила сравнивается с силой инерции.

Рассмотрим нашу задачу о вынужденных колебаниях груза, которая описывается уравнением (1). В этом уравнении четыре слагаемых, и каждое слагаемое есть сила (2). Сравним силы сопротивления и инерции. Для подобия колебаний модели (с индексом 1) и натуры (без индекса) отношения этих сил должны быть одинаковыми:

$$
\frac{F_{k 1}}{F_{k}}=\frac{F_{m 1}}{F_{m}}, \quad \frac{k_{1} \dot{x}_{1}}{k \dot{x}}=\frac{m_{1} \ddot{x}_{1}}{m \ddot{x}}, \quad \frac{k_{1}}{k} \frac{\dot{x}_{1}}{\dot{x}}=\frac{m_{1}}{m} \frac{\ddot{x}_{1}}{\ddot{x}} .
$$

С учетом значений параметров модели (10) получаем первый индикатор подобия, а из него по (13) - число подобия $\pi_{1}$ :

$$
k_{c} \frac{x_{c}}{t_{c}}=m_{c} \frac{x_{c}}{t_{c}^{2}}, \quad \frac{k_{c} x_{c}}{t_{c}} \frac{t_{c}^{2}}{m_{c} x_{c}}=1, \quad \lambda_{1}=\frac{k_{c} t_{c}}{m_{c}}=1, \quad \pi_{1}=\frac{k t}{m} .
$$

Проводя аналогичные сравнения силы упругости и силы инерции, а также внешней силы и силы инерции, найдем остальные числа подобия:

$$
\begin{gathered}
\frac{F_{c 1}}{F_{c}}=\frac{F_{m 1}}{F_{m}}, \quad \frac{c_{1}}{c} \frac{x_{1}}{x}=\frac{m_{1}}{m} \frac{\ddot{x}_{1}}{\ddot{x}}, \quad \lambda_{2}=\frac{c_{c} t_{c}^{2}}{m_{c}}=1, \quad \pi_{2}=\frac{c t^{2}}{m} \\
\frac{F_{\omega 1}}{F_{\omega}}=\frac{F_{m 1}}{F_{m}}, \quad \frac{P_{1}}{P} \frac{\sin \left(\omega_{1} t_{1}\right)}{\sin (\omega t)}=\frac{m_{1}}{m} \frac{\ddot{x}_{1}}{\ddot{x}}, \quad \omega_{1} t_{1}=\omega t, \quad \pi_{4}=\omega t ;
\end{gathered}
$$




$$
\lambda_{3}=\frac{P_{c} t_{c}^{2}}{m_{c} x_{c}}=1, \quad \pi_{3}=\frac{P t^{2}}{m x} .
$$

Таким образом, видим, что этот способ приводит к тем же числам подобия (8).

K. С. Басниев и др. [6] для выяснения физического смысла чисел подобия рассматривают в жидкости параллелепипед. На него действуют сила тяжести, сила локальной инерции, сила конвективной инерции, сила трения. Далее показывают, что числа подобия есть отношения соответствующих сил к силе инерции.

Аналогично действуют П. М. Алабужев и др. [2].

В. А. Винников и Г. Г. Каркашадзе [10], а также В. П. Корпачев [19] выводят основные числа подобия в жидкости из соотношения сил:

- составляя отношение силы тяжести к силе инерции, получает число подобия Фруда;

- в случае, когда на жидкость действуют только силы вязкого трения и влиянием сил тяжести можно пренебречь, из отношения сил инерции к силам трения получается число подобия Рейнольдса;

- из отношения параметра давления к удвоенной величине динамического (скоростного) напора получается число подобия Эйлера.

А. А. Кудинов [22] при выводе чисел подобия использует способ двух уравнений - для натуры и модели, получает индикатор, а затем число подобия Ньютона как отношение внешней силы к силе инерции. Затем рассматривает действие отдельных сил:

- если внешняя сила является силой тяжести, то из числа Ньютона получается число Фруда;

- если внешняя сила является силой давления, то получается число Эйлера;

- если внешняя сила является силой внутреннего трения, то получается число Рейнольдса.

А. К. Мартынов [27], а также А. М. Мхитарян [30] выводят используемые в аэродинамике числа подобия Эйлера, Рейнольдса, Фруда, Маха, Струхаля как отношения соответствующих сил к силе инерции.

При использовании способа Д5 нужно быть осторожным и не забывать, что этот способ является следствием более общего способа Д4, в противном случае можно сделать неверное заключение. В частности, в учебнике Б. М. Яворского и А. А. Пинского [47, с. 107] при рассмотрении задачи о движении тела в жидкости сначала через анализ размерностей выводятся выражения для двух сил-для силы сопротивления $R$, возникающей вследствие разности давлений на передней и задней кромках обтекаемого тела, и для силы вязкого трения $T$. Затем составляется отношение $R / T$ и получается выражение, совпадающее с числом Рейнольдса. Отсюда авторы делают вывод, что число Рейнольдса есть отношение сопротивления давления $R$ к сопротивлению трения $T$. Такое толкование физического смысла числа Рейнольдса противоречит общепринятому, которое следует из безразмерного уравнения это отношение сил инерции к силам сопротивления трения за счет вязкости. 


\section{2. Способы вывода критериев подобия из анализа размерностей}

Решения задач методом анализа размерностей основано на второй, или Пи-теореме. Такое название, происходящее от традиционного обозначения безразмерных комбинаций с помощью прописной или строчной греческой буквы «пи», используется в русскоязычной литературе. В англоязычной литературе теорему обычно связывают с именем Букингема (E. Buckingham), а во франкоязычной - с именем Ваши (A. Vaschy) $)^{2}$.

Здесь рассмотрим следующие семь способов вывода чисел подобия из анализа размерностей, которые для краткости обозначим через P1-P7:

P1. Способ комбинации переменных, или способ непосредственных рассуждений, или способ Бертрана;

Р2. Ранние способы Аппелля, Федермана, Толмэна, Ипсена;

Р3. Способ преобразования формул размерностей в степенные комплексы;

P4. Способ частичных комплексов, или способ предварительной группировки, или способ нулевых размерностей, или способ Букингема;

Р5. Способ полного двустороннего комплекса, или способ Рэлея;

Р6. Способ полного одностороннего комплекса, или способ глобального критерия;

P7. Способ качественного физико-математического анализа, или способ Морозова.

Отметим, что приведенная терминология в названиях способов не устоялась и в разных источниках названия методов могут отличаться. Общие положения по выводу чисел подобия будем иллюстрировать примерами для задачи о вынужденных колебаниях груза, которые описываются уравнением (1). В безразмерном виде задача сводится к уравнению (7) и четырем критериям подобия (8). Покажем, что те же числа подобия можно вывести через анализ размерностей, не обращаясь к уравнению колебаний.

P1. Способ комбинации переменных, или способ непосредственных рассуждений, или способ Бертрана. В уравнение колебаний (1) входит семь $(n=7)$ размерных параметров

$$
m, \quad x, \quad t, \quad P, \quad \omega, \quad c, \quad k,
$$

размерности которых в классе $L M T$ известны:

$$
\begin{gathered}
{[m]=M, \quad[x]=L, \quad[t]=T, \quad[P]=M L T^{-2},} \\
{[\omega]=T^{-1}, \quad[c]=M T^{-2}, \quad[k]=M T^{-1} .}
\end{gathered}
$$

Здесь три $(k=3)$ независимые размерности - $L, M, T$. В соответствии с Питеоремой задачу можно описать с помощью трех размерных и четырех ( $p=$ $=n-k=7-3=4)$ безразмерных параметров.

Величины $m, x, t$ имеют простейшие размерности - килограмм, метр и секунда, которые невозможно свести к безразмерному виду. Остались величины

$$
k, \quad c, \quad P, \quad \omega .
$$

\footnotetext{
${ }^{2}$ По материалам сайта Wikipedia.
} 
Рассмотрим коэффициент демпфирования $k$. Поскольку его размерность есть $M / T$, то для создания безразмерного комплекса его нужно умножить на время и поделить на массу:

$$
[k]=\frac{M}{T}, \quad \pi_{1}=k \frac{t}{m}, \quad\left[\pi_{1}\right]=\frac{M}{T} \frac{T}{M}=1 .
$$

Рассмотрим коэффициент упругости пружины $c$. Поскольку его размерность есть $M / T^{2}$, для создания безразмерного комплекса его нужно умножить на время в квадрате и поделить на массу:

$$
[c]=\frac{M}{T^{2}}, \quad \pi_{2}=c \frac{t^{2}}{m}, \quad\left[\pi_{2}\right]=\frac{M}{T^{2}} \frac{T^{2}}{M}=1 .
$$

Точно так же составляем безразмерные комбинации для амплитуды $P$ и круговой частоты $\omega$ возмущающей силы:

$$
\begin{gathered}
{[P]=\frac{L M}{T^{2}}, \quad \pi_{3}=P \frac{t^{2}}{m x}, \quad\left[\pi_{3}\right]=\frac{L M}{T^{2}} \frac{T^{2}}{M L}=1 ;} \\
{[\omega]=\frac{1}{T}, \quad \pi_{4}=\omega t, \quad\left[\pi_{4}\right]=\frac{1}{T} T=1 .}
\end{gathered}
$$

В результате приходим к тем же критериям подобия (8).

Способ Р1 описан под разными названиями у многих авторов и применялся для различных задач.

П. М. Алабужев и др. $[1,2]$ называют этот способ методом анализа размерностей.

П. В. Бриджмен [8] этим способом решает задачу о периоде колебаний маятника (с. 12), задачу Рэлея 1915 года о колебаниях капли жидкости в невесомости под влиянием сил поверхностного натяжения (с. 14), задачу о жесткости прогибающейся балки (с. 78).

Г. И. Баренблатт $[4,5]$ рассматривает свою работу как продолжение книги П. Бриджмена [8] и способ Р1 применяет в задачах для периода колебаний математического маятника, для поступательного движения шара в газе с большой скоростью, для доказательства теоремы Пифагора (с. 55). Этим способом Г. И. Баренблатт выводит критерии Рейнольдса (с. 62) и Фруда (c. 64$)$, из задачи о конвективном теплообмене в горизонтальном слое жидкости (с. 71) выводит критерии Грасгофа, Рэлея и Прандтля.

В. А. Веников [9] называет этот способ методом непосредственных рассуждений (с. 92) и, применяя его к задаче о теплоотдаче от круглой трубы к поперечно омывающему ее потоку жидкости, получает критерии Нуссельта и Рейнольдса.

М. В. Кирпичев [14] способ Р1 называет способом Бертрана (с. 75) и отмечает, что суть его состоит в попытках комбинировать между собою размерные величины так, чтобы получились безразмерные произведения. Как отмечает М. В. Кирпичев, «руководящей нитью в таком рассуждении являются чутье и догадка».

С. С. Кутателадзе [23] при рассмотрении сложных задач гидромеханики с учетом влияния температуры и массопереноса формирует безразмерные комплексы путем подбора размерных величин. 
Л. Г. Лойцянский [24-26] применяет способ рассуждений при рассмотрении различных задач гидромеханики. Разделяя полученные безразмерные комплексы на критерии подобия и числа подобия (функции от критериев), Л. Г. Лойцянский показывает, что можно в некоторых задачах предсказать вид решения и перейти от уравнений в частных производных к обыкновенным дифференциальным уравнениям, что позволяет найти решение задачи в замкнутом виде.

Л. И. Седов [37] применяет этот способ к решению следующих задач:

- о периоде колебаний математического маятника (с. 36);

- об истечении весомой жидкости (с. 39);

- о движении жидкости в трубах (с. 40);

- о движении тела в жидкости (с. 46);

- о теплоотдаче тела в потоке жидкости с упоминанием решения Рэлея и парадокса Рябушинского (с. 53);

- о равновесии упругих конструкций с моделированием на центрифуге и выводом соотношений между внешней нагрузкой и собственным весом (с. 60$)$;

- об установившемся (с. 67) и неустановившемся (с. 71) движении твердого тела в сжимаемой жидкости;

- о движении корабля с обоснованием удешевления перевозок для больших судов и самолетов (с. 76);

- о глиссировании по поверхности воды (с. 84);

- об ударе о воду, о рикошете, о посадке гидросамолета (с. 91);

- о вертикальном ударе о воду (с. 96);

- о погружении клина в воду (с. 99).

В. А. Соколов [41] показывает применение этого способа на примерах для задач, возникающих в нефтегазодобыче.

P2. Ранние способы Аппелля, Федермана, Толмэна, Ипсена. K этой группе способов вывода критериев подобия из анализа размерностей отнесем такие способы, которые упоминаются в литературе, но широкого применения не получили. В силу громоздкости и сложности действий по этим способам, а также их непопулярности, мы их применять к задаче о колебаниях груза не будем.

М. В. Кирпичев [14] описывает три ранних способа: Аппелля [48,49] 1893 г., Федермана [42] 1911 г., Толмэна [56-58] 1914 г. Применение способа Аппелля показано на примере отыскания периода колебаний математического маятника. Способ Федермана описан на примере отыскания скорости истечения жидкости через отверстие, сделанное в сосуде на известной глубине. Способ Толмэна описан на примере вывода закона состояния для идеального газа.

Х. Шенк [45] описывает способ последовательного исключения размерностей, или поэтапный способ Ипсена, и его модификацию - способ линейных пропорциональностей Барра. При этом Х. Шенк отмечает, что этот способ «не так прост, особенно при большом числе переменных и при наличии 4-5 основных размерностей».

Ю. А. Дьячков и М. А. Черемшанов [12] показывают применение способов Ипсена и Барра к задаче движения автомобильного амортизатора. 
Р3. Способ преобразования формул размерностей в степенные комплексы. Рассмотрим нашу задачу о колебаниях груза. Для каждой величины заменим ее размерность (15) соответствующими параметрами и разделим каждое равенство на его правую часть:

$$
\begin{gathered}
\frac{m}{m}=1, \quad \frac{x}{x}=1, \quad \frac{t}{t}=1, \quad \frac{k}{m t^{-1}}=\frac{k t}{m}=\pi_{1}, \\
\frac{c}{m t^{-2}}=\frac{c t^{2}}{m}=\pi_{2}, \quad \frac{P}{x m t^{-2}}=\frac{P t^{2}}{m x}=\pi_{3}, \quad \frac{\omega}{t^{-1}}=\omega t=\pi_{4} .
\end{gathered}
$$

Опять получаем те же критерии подобия (8).

Этот способ описывает А. А. Гухман [11]. Он показывает его применение в задаче о гидродинамическом сопротивлении при стационарном течении несжимаемой жидкости по каналу-трубе (с. 280). В этой задаче получает критерии Эйлера и Рейнольдса.

P4. Способ частичных комплексов, или способ предварительной группировки, или способ нулевых размерностей, или способ Букингема. В задачу о вынужденных колебаниях груза входят 7 размерных величин (14), которые имеют размерности (15). По способу Букингема «из 7 размерных величин выделяются 3 первичных и ищут 4 критерия, содержащих каждый по одной вторичной величине, подбирая для них комбинацию первичных, образующих безразмерный комплекс» [15].

Выберем в качестве первичных, или основных, параметры $m, x, t$, которые образуют базовую тройку, т. к. содержат все три независимые размерности задачи - килограмм, метр и секунду $(M, L, T)$. Будем добавлять по одному размерному параметру к базовой тройке и формировать безразмерный комплекс.

1. Параметры $(m, x, t)+c$. Формируем безразмерный комплекс в виде

$$
\pi_{41}=\frac{c}{m^{a} x^{b} t^{d}} .
$$

Эта же формула в размерностях:

$$
M^{0} L^{0} T^{0}=\left[\pi_{41}\right]=\frac{M T^{-2}}{M^{a} L^{b} T^{d}}=M^{1-a} L^{-b} T^{-2-d} .
$$

Уравнивая показатели степеней в левой и правой частях уравнения, получаем систему линейных алгебраических уравнений относительно показателей:

$$
M: 1-a=0, \quad L:-b=0, \quad T:-2-d=0 .
$$

Разрешаем эту систему уравнений:

$$
a=1, \quad b=0, \quad d=-2
$$

и получаем уже знакомый критерий подобия $\pi_{2}$ :

$$
\pi_{41}=\frac{c}{m^{1} x^{0} t^{-2}}=\frac{c t^{2}}{m}=\pi_{2} .
$$


2. Параметры $(m, x, t)+P$. Формируем безразмерный комплекс в виде

$$
\pi_{42}=\frac{P}{m^{a} x^{b} t^{d}}
$$

Записываем эту формулу в размерностях, уравниваем показатели степеней слева и справа, находим показатели степеней:

$$
a=1, \quad b=1, \quad d=-2 .
$$

Подставляя найденные значения в исходное выражение, получаем уже знакомый критерий подобия $\pi_{3}$ :

$$
\pi_{42}=\frac{P}{m^{1} x^{1} t^{-2}}=\frac{P t^{2}}{m x}=\pi_{3} .
$$

3. Параметры $(m, x, t)+\omega$. Формируем безразмерный комплекс в виде

$$
\pi_{43}=\frac{\omega}{m^{a} x^{b} t^{d}}
$$

Действуя точно так же, как и в предыдущих случаях, находим

$$
\begin{gathered}
a=0, \quad b=0, \quad d=-1 \\
\pi_{43}=\frac{\omega}{m^{0} x^{0} t^{-1}}=\omega t=\pi_{4} .
\end{gathered}
$$

4. Параметры $(m, x, t)+k$. Формируем безразмерный комплекс в виде

$$
\pi_{44}=\frac{k}{m^{a} x^{b} t^{d}} .
$$

Аналогичные действия дают следующий результат:

$$
\begin{gathered}
a=1, \quad b=0, \quad d=-1 ; \\
\pi_{44}=\frac{k}{m^{1} x^{0} t^{-1}}=\frac{k t}{m}=\pi_{1} .
\end{gathered}
$$

В результате получили те же четыре критерия (8).

Этот способ основан на выводе теоремы Федермана-Букингема (Пи-теоремы, 1914 г.) и предложен Букингемом [50].

П. М. Алабужев и др. [1] называют этот способ методом нулевых размерностей и показывают его применение на примере вынужденных колебаний груза. В [2] приводится применение этого способа для многочисленных задач из различных отраслей техники.

Г. И. Баренблатт [4,5] представляет способ Р4 как следствие доказательства Пи-теоремы (с. 15, 44). Этим способом решает задачу о распределении скорости в пристеночной области сдвигового турбулентного потока (с. 115), задачу для расплывания грунтовых вод (с. 126). 
П. В. Бриджмен [8] решает этим способом задачу о периоде колебаний ящика с жидкостью (с. 69), задачу Стокса о скорости падающей сферы в жидкости (с. 75), задачу о давлении идеального газа (с. 80).

В. А. Веников [9] отмечает, что этот способ вытекает из Пи-теоремы Букингема, показывает его применение на примере вынужденных колебаний груза (с. 73), а также на задаче о падении давления при движении вязкой жидкости в трубе, из которой получает критерии Рейнольдса и Эйлера (с. 90).

Ю. А. Дьячков и М. А. Черемшанов [12] называют этот способ способом Букингема и показывают его применение на примере движения автомобильного амортизатора.

M. В. Кирпичев [14] также называет этот способ способом Букингема, т. к. он вытекает из Пи-теоремы Букингема, и показывает его применение на примере теплопередачи между стенкой трубки и потоком жидкости.

С. Д. Корнеев [18] и С. И. Пинчук [33] упоминают этот способ, но примеров не приводят.

О. Я. Романов и В. В. Ходосов [35] способ Р4 называют способом предварительной группировки и показывают его применение на задаче о движении капли жидкости в потоке вязкого газа при изотермических условиях, в которой получаются два независимых критерия - Вебера и Рейнольдса.

Р. Х. Санников [36] при описании способов получения критериев подобия на основе Пи-теоремы выделяет классический способ (в нашем случае способ Р6) и видоизмененный классический способ (в нашем случае способ P4). Применение этих способов показывает на примере для вынужденных колебаний груза.

Р5. Способ полного двустороннего комплекса, или способ Рэлея. В задачу о вынужденных колебаниях груза входит 7 величин (14). Допустим, необходимо найти коэффициент силы сопротивления $k$, который может зависеть от всех остальных параметров задачи. Составим полный двусторонний комплекс в виде

$$
k=C_{0} m^{u} x^{v} t^{w} c^{q} P^{r} \omega^{s},
$$

где $C_{0}$ - безразмерный коэффициент. Перепишем уравнение (16) в размерностях (15):

$$
\begin{gathered}
M T^{-1}=M^{u} L^{v} T^{w}\left(M T^{-2}\right)^{q}\left(L M T^{-2}\right)^{r}\left(T^{-1}\right)^{s}, \\
M T^{-1}=M^{u+q+r} L^{v+r} T^{w-2 q-2 r-s} .
\end{gathered}
$$

Поскольку размерности левой и правой частей этого уравнения должны быть одинаковыми, получим следующую систему линейных алгебраических уравнений (СЛАУ):

$$
M: 1=u+q+r, \quad L: 0=v+r, \quad T:-1=w-2 q-2 r-s .
$$

Из трех уравнений (17) можно определить только три неизвестных. Будем искать $u, v, w$. Тогда показатели степеней $q, r, s$ будут считаться независимыми:

$$
u=1-q-r, \quad v=-r, \quad w=-1+2 q+2 r+s .
$$

Подставляя полученные значения в исходное уравнение (16), получим

$$
k=C_{0} m^{1-q-r} x^{-r} t^{-1+2 q+2 r+s} c^{q} P^{r} \omega^{s} .
$$


Группируем сомножители по степеням

$$
k=C_{0}\left(m^{1} t^{-1}\right)\left(m^{-1} c t^{2}\right)^{q}\left(m^{-1} x^{-1} t^{2} P\right)^{r}(t \omega)^{s},
$$

и в окончательном виде получаем

$$
\left(\frac{k t}{m}\right)=C_{0}\left(\frac{c t^{2}}{m}\right)^{q}\left(\frac{P t^{2}}{m x}\right)^{r}(\omega t)^{s} .
$$

Безразмерные комплексы в скобках дают те же четыре критерия подобия (8).

Классическая форма записи искомой функции по способу Рэлея имеет вид (16). Однако некоторые авторы используют модифицированную запись, в которой слева стоит не искомая величина, а константа

$$
C_{0}=k m^{u 1} x^{v 1} t^{w 1} c^{q 1} P^{r 1} \omega^{s 1} .
$$

Отличие последней записи от (16) только в знаках показателей степеней. Поэтому делать различия в классической и модифицированной записях способа Рэлея мы не будем.

Сам Рэлей разрабатывал свой способ вывода критериев подобия в 1900-х годах и применял его к различным задачам, которые до сих пор упоминаются как задачи Рэлея [53].

В. А. Архипов и А. П. Березиков [3] показывают применение этого способа на задаче о теплообмене при стационарном турбулентном течении теплоносителя (газа или жидкости) в трубе.

Г. Биркгоф [7] использует способ Р5 при определении силы сопротивления жидкости в зависимости от ее плотности, а также скорости и диаметра движущегося тела (с. 125).

П. В. Бриджмен [8] решает этим способом задачу о периоде вращения двух масс (с. 16), две задачи Рэлея - о скорости переноса тепла в жидкости (с. 19) и о скорости волны в жидкости (с. 66).

М. В. Кирпичев [14] определяет этот способ как метод Рэлея (с. 81) и показывает его использование на примере теплоотдачи.

А. А. Кудинов [22] рассматривает анализ размерностей по способам Р5 и Р6. Способом Р5, который называет методом Рэлея, получает критерии подобия Фруда, Эйлера, Рейнольдса, Струхаля и делает вывод, что анализ уравнений и анализ размерностей являются, по существу, разными методами одной и той же системы исследования, основанной на использовании обобщенных безразмерных переменных.

С. И. Пинчук [33] упоминает этот способ, но примеров не приводит.

Л. И. Седов [37] способом Р5 решает задачу о малых волнах на поверхности жидкости (с. 101).

Г. Хантли [43] приводит решение этим способом 45 задач из области механики твердых тел, гидромеханики, теплопередачи, электричества.

Х. Шенк [45] описывает способ Р5 как рэлеевский метод решения на основании теоремы Букингема.

Л. А. Эпштейн [46] использует способ Рэлея в модифицированном виде в различных задачах гидромеханики. 
P6. Способ полного одностороннего комплекса, или способ глобального критерия. Рассмотрим нашу задачу о вынужденных колебаниях груза. Составим глобальный критерий подобия, включающий все параметры задачи (14):

$$
\pi=m^{u} x^{v} t^{w} c^{q} P^{r} \omega^{s} k^{h}
$$

Перепишем уравнение (18) в размерностях:

$$
\begin{gathered}
1=M^{u} L^{v} T^{w}\left(M T^{-2}\right)^{q}\left(L M T^{-2}\right)^{r}\left(T^{-1}\right)^{s}\left(M T^{-1}\right)^{h}, \\
1=M^{u+q+r+h} L^{v+r} T^{w-2 q-2 r-s-h} .
\end{gathered}
$$

Уравнивая размерности слева и справа, получим следующую СЛАУ:

$$
u+q+r+h=0, \quad v+r=0, \quad w-2 q-2 r-s-h=0 .
$$

Из трех уравнений можем определить три неизвестных, остальные 4 можем задать произвольно. Выберем в качестве базовых три параметра $m, x$, $t$, размерности которых содержат все три независимые размерности задачи $M, L, T$. Тогда соответствующие показатели степеней $u, v, w$ находятся из СЛАУ (19), а остальные четыре показателя $q, r, s, h$ для параметров $c, P$, $\omega, k$ задаются произвольно. Перепишем СЛАУ (19) так, чтобы показатели степеней $u, v, w$ находились в левой части:

$$
u=-q-r-h, \quad v=-r, \quad w=2 q+2 r+s+h .
$$

В СЛАУ (20) справа стоят 4 независимых показателя $q, r, s, h$. Поочередно будем задавать одному из них значение 1 , а остальным - значение 0 . Всего наберется 4 комбинации.

Комбинация 1. Примем $q=1, r=s=h=0$. В соответствии с (18) это равносильно тому, что к базовой тройке параметров $m, x, t$ добавляется четвертый параметр $c$. Если сравнить эту комбинацию со способом Р4, то она эквивалентна формированию безразмерного комплекса $\pi_{41}$. Тогда из (20) получим

$$
u=-1, \quad v=0, \quad w=2 .
$$

Подставляем эти значения в (18) и находим, что такому набору показателей соответствует знакомый критерий подобия $\pi_{2}$ :

$$
\pi=m^{-1} x^{0} t^{2} c^{1} P^{0} \omega^{0} k^{0}=\frac{c t^{2}}{m}=\pi_{2} .
$$

Комбинация 2. Примем $q=0, r=1, s=h=0$. В соответствии с (18) это равносильно тому, что к базовой тройке параметров $m, x, t$ добавляется четвертый параметр $P$. Такая комбинация эквивалентна формированию безразмерного комплекса $\pi_{42}$ в способе Р4. Решая СЛАУ (20), получим

$$
u=-1, \quad v=-1, \quad w=2 .
$$

Подставляя эти значения в (18), находим критерий подобия $\pi_{3}$ :

$$
\pi=m^{-1} x^{-1} t^{2} c^{0} P^{1} \omega^{0} k^{0}=\frac{P t^{2}}{m x}=\pi_{3} .
$$


Комбинация 3. Примем $q=r=0, s=1, h=0$. Действуя, как и в предыдущих случаях, находим

$$
\begin{gathered}
u=0, \quad v=0, \quad w=1, \\
\pi=m^{0} x^{0} t^{1} c^{0} P^{0} \omega^{1} k^{0}=\omega t=\pi_{4} .
\end{gathered}
$$

Комбинация 4. Примем $q=r=s=0, h=1$. Аналогичные действия дают

$$
\begin{gathered}
u=-1, \quad v=0, \quad w=1, \\
\pi=m^{-1} x^{0} t^{1} c^{0} P^{0} \omega^{0} k^{1}=\frac{k t}{m}=\pi_{1} .
\end{gathered}
$$

В результате получили те же четыре критерия подобия (8). Сопоставляя процедуры действия данного способа со способом Р4, видим, что способ глобального критерия является обобщением способа Букингема.

П. М. Алабужев и др. [1] показывают применение этого способа на примере вынужденных колебаний груза.

А. А. Кудинов [22] получает этим способом критерии подобия Фруда, Эйлера, Рейнольдса, Струхаля.

С. И. Пинчук [33] упоминает этот способ, но примеров не приводит.

О. Я. Романов и В. В. Ходосов [35] называют этот способ способом глобального критерия и показывают его применение в задаче по определению силы сопротивления движению твердого тела в несжимаемой жидкости, в которой из 7 размерных параметров, имеющих 3 независимых размерности, следуют 4 критерия подобия - коэффициент силы сопротивления, угол атаки в радианах, число Рейнольдса и число Фруда.

P. Х. Санников [36] называет этот способ классическим способом анализа размерностей и показывает его применение на примере для вынужденных колебаний груза.

Л. А. Шаповалов [44] применяет этот способ для составления проекта проведения работ по моделированию прочности и жесткости элементов конструкций в различных задачах.

P7. Способ качественного физико-математического анализа, или способ Морозова. Все ранее описанные способы опирались на Пи-теорему. Обязательным условием их применения было наличие полного списка всех размерных и безразмерных величин, участвующих в задаче. В отличие от них способ Морозова не требует знания всех величин задачи. Подход Морозова похож на способ Рэлея - также ищется одна неизвестная величина в виде алгебраического степенного комплекса, составленного из остальных величин. Но в отличие от способа Рэлея у Морозова в исходной формуле участвуют не все параметры задачи. Так же, как и в способе подбора, здесь необходимо правильно угадать исходную зависимость искомой функции от параметров задачи.

Н. А. Морозов разрабатывал свой способ в 1890-1906 годах, опубликовал его в 1908 году [29], задолго до появления Пи-теоремы Федермана-Букингема (1914г.). Поэтому в его изложении отсутствует понятие безразмерного критерия подобия. Морозов не различает размерную величину и ее размерность, и то и другое обозначает одной буквой. Из-за этого трудно понять, о чем конкретно идет речь. По способу Морозова невозможно получить 


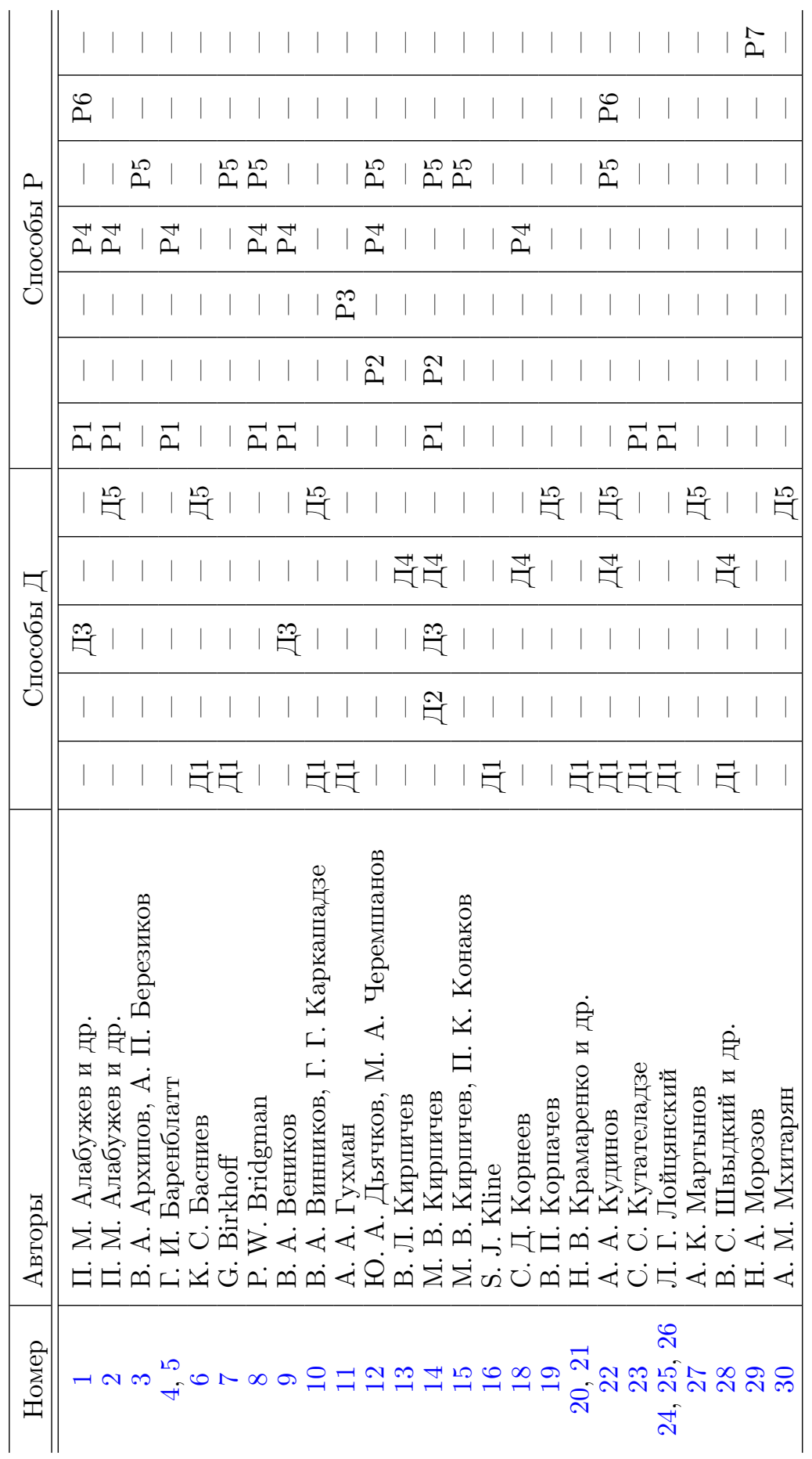




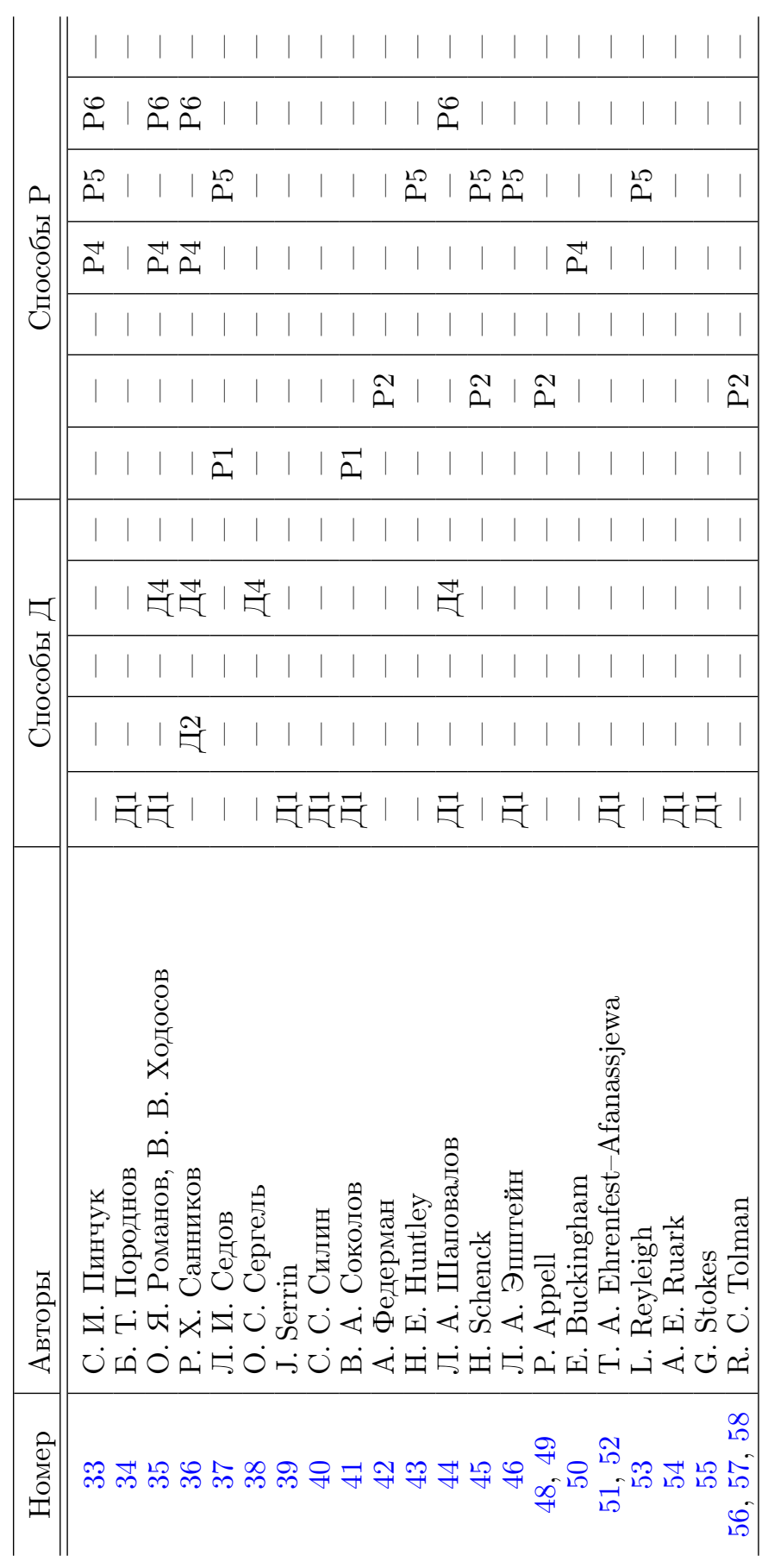


несколько критериев подобия, как это имеет место в нашей задаче о вынужденных колебаниях груза.

В наше время такой подход не используется. С. С. Кутателадзе [23] называет его «несколько наивным». Здесь этот способ упоминается для подчеркивания исторического приоритета российского ученого Н. А. Морозова в разработке анализа размерностей. Для знакомства с этим способом можно рекомендовать обратиться к первоисточнику [29], в котором автор показывает применение своего способа к задаче о собственных колебаниях струны.

\section{Заключение}

На основании рассмотрения одной задачи о колебаниях груза видим, что одни и те же числа подобия можно получить как из анализа уравнений, так и из анализа размерностей. Приведем сводную таблицу использования авторами цитируемых работ перечисленных способов вывода критериев подобия. Из этой таблицы видно, что наиболее употребительными являются следующие способы:

Д1. Способ нормализации уравнения, или приведения уравнения к безразмерному виду;

Д4. Способ сравнения двух уравнений через индикаторы подобия;

P4. Способ частичных комплексов, или способ предварительной группировки, или способ нулевых размерностей, или способ Букингема;

Р5. Способ полного двустороннего комплекса, или способ Рэлея;

Р6. Способ полного одностороннего комплекса, или способ глобального критерия.

В дополнение к перечисленным общепризнанным способам хочется отметить изящный способ А. А. Гухмана Р3, который в силу своей простоты и скорости достижения результата можно назвать «экспресс-способом».

В заключение добавим, что получить доступ ко многим классическим источникам по теории подобия, а также прочитать ценные комментарии к ним можно на веб-странице [31].

Конкурирующие интересы. Я заявляю об отсутствии явных и потенциальных конфликтов интересов, связанных с публикацией настоящей статьи.

Авторский вклад и ответственность. Я несу полную ответственность за предоставление окончательной версии рукописи в печать. Окончательная версия рукописи мною одобрена

Финансирование. Исследование выполнялось без финансирования.

\section{Библиографический список}

1. Алабужев П. М., Геронимус В. В., Минкевич Л. М., Шеховцов Б. А. Теория подобия и размерностей. Моделирование. М.: Высш. шк., 1968. 206 с.

2. Алабужев П. М., Кирнарский М. Ш., Полищук В. Г., Соколов В. С., Чижов А. Е., Юшин В. В. Основы теории подобия, размерности, моделирования. Курск: Курск. политехнич. ин-т, 1993. $103 \mathrm{c.}$

3. Архипов В. А., Березиков А. П. Основы теории инженерно-физического эксперименma. Томск: Томск политехнич. ун-т, 2008. 206 с.

4. Баренблатт Г. И. Подобие, автомодельность, промежуточная асимптотика. Теория и приложения к геофизической гидродинамике. Л.: Гидрометеоиздат, 1982. 256 с.

5. Баренблатт Г. И. Анализ размерностей. М.: МФТИ, 1987. 168 с. 
6. Басниев К. С., Дмитриев Н. М., Розенберг Г. Д. Нефтегазовая гидромеханика. М., Ижевск: Инст. комп. исслед., 2005. 544 с.

7. Биркгоф Г. Гидродинамика. Методы, факты, подобие. М.: Иностр. лит-ра, 1963. 246 с.

8. Бриджмен П. В. Анализ размерностей. Л.-М.: ОНТИ ГТТИ, 1934. 119 с.; Ижевск: Регулярная и хаотическая динамика, 2001. 148 с.

9. Веников В. А. Теория подобия и моделирования (применительно к задачам электроэнергетики). М.: Высш. шк., 1976. 479 с.

10. Винников В. А., Каркашадзе Г. Г. Гидромеханика. М.: Моск. госуд. горн. ун-т, 2003. $302 \mathrm{c}$.

11. Гухман А. А. Введение в теорию подобия. М.: Высш. шк., 1973. 296 с.

12. Дьячков Ю. А., Черемшанов М. А. Моделирование систем автомобилестроения. Пенза: ПГУ, 2009. 240 с.

13. Кирпичев В. Л. Беседы о механике. М., Л.: ГИТТЛ, 1950. 360 с.

14. Кирпичев М. В. Теория подобия. М.: АН СССР, 1953. 96 с.

15. Кирпичев М. В., Конаков П. К. Математические основы теории подобия. М.: АН CCCP, 1949. $106 \mathrm{c}$.

16. Клайн С. Дж. Подобие и приближенные методы. М.: Мир, 1968. 302 с.

17. Коган И. Ш. О желательности расширения масштабов применения теории подобия при преподавании технических наук / Машиностроение. Конструкиии и технологии: Сб. научных тр. Т. 3. Киров: ВятГТУ, 1998. С. 64-69.

18. Корнеев С. Д. Гидрогазодинамика. М.: МГИУ, 2011. 230 с.

19. Корпачев В. П. Теоретические основы водного транспорта леса. М.: Акад. естествознания, 2009. 236 с.

20. Крамаренко Н. В., Ситнов К. В. Теория подобия и спецэффекты в кино / Наука. Промышленность. Оборона: Тр. 15 Всерос. науч.-техн. конф. (Новосибирск, 23-25 апреля 2014 г.). Новосибирск: НГТУ, 2014. С. 347-351.

21. Крамаренко Н. В., Капустина А. А., Лаврина В. М., Скоробогатова А. А. Использование теории подобия для проведения инженерного эксперимента / Наука. Промыиленность. Оборона: Тр. 20 Всерос. науч.-техн. конф. Т. 1 (Новосибирск, 17-19 апреля 2019 г.). Новосибирск: НГТУ, 2019. С. 96-101.

22. Кудинов А. А. Техническая гидромеханика. Самара: СамГТУ, 2006. 295 с.

23. Кутателадзе С. С. Анализ подобия и физические модели. Новосибирск: Наука, 1986. 296 с.

24. Лойцянский Л. Г. Механика жидкости и газа. М.: Наука, 1970. 904 с.

25. Лойцянский Л. Г. Механика жидкости и газа. М.: Дрофа, 2003. 840 с.

26. Лойцянский Л. Г. Методы подобия и размерностей в механике жидкости и газа / Сб. метод. статей по теорет. механике, Вып. 11. М.: Высш. шк., 1981. 22-31 с.

27. Мартынов А. К. Прикладная аэродинамика. М.: Машиностроение, 1972. 448 с.

28. Швыдкой В. С. (ред.) Механика жидкости и газа. М.: Академкнига, 2003. 464 с.

29. Морозов Н. А. Основы качественного физико-математического анализа и новые физические факторы, обнаруживаемые им в различных явлениях природы. М., 1908. 402 с.

30. Мхитарян А. М. Аэродинамика. М.: Машиностроение, 1976. 448 с.

31. Некоторые обзорные работы и первоисточники по истории пи-теоремы и теории подобия. Режим доступа: http://gidropraktikum.narod.ru/pi-theorem-history.htm (дата обращения: 29.03.2021).

32. Основы теории подобия и моделирования. Терминология. Сборник рекомендуемых терминов / Вып. 88. М.: Наука, 1973. 50 с.

33. Пинчук С. И. Организачия эксперимента при моделировании и оптимизации технических систем. Днепропетровск: Дива, 2008. 248 с.

34. Породнов Б. Т. Численные методы решения задач механики сплошных сред. Екатеринбург: УГТУ-УПИ, 2008. 
35. Романов О. Я., Ходосов В. В. Моделирование при проектировании сложных технических систем. СПб.: Балт. гос. техн. ун-т, 2006. 60 с.

36. Санников Р. Х. Теория подобия и моделирования. Планирование инженерного эксперимента. Уфа: УГНТУ, 2010. 253 с.

37. Седов Л. И. Методы подобия и размерности в механике. М.: Наука, 1977. 440 с.

38. Сергель О. С. Прикладная гидрогазодинамика. М.: Машиностроение, 1981. 374 с.

39. Серрин Дж. Математические основы классической механики жидкости. М.: Иностр. лит-ра, 1963. 256 с.

40. Силин С. С. Метод подобия при резании материалов. М.: Машиностроение, 1979. 152 с.

41. Соколов В. А. Основы теории подобия и анализа размерностей в небтегазодобыче. Ухта: УГТУ, 2001. 159 с.

42. Федерман А. О некоторых общих методах интегрирования уравнений с частными производными первого порядка // Известия Санкт-Петербургского политехнического института императора Петра Великого. Отдел техники, естествознания и математики, 1911. Т. 16, № 1. С. 97-155.

43. Хантли Г. Анализ размерностей. М.: Мир, 1970. 176 с.

44. Шаповалов Л. А. Моделирование в задачах механики элементов конструкиий. М.: Машиностроение, 1990. 288 с.

45. Шенк Х. Теория инженерного эксперимента. М.: Мир, 1972. 384 с.

46. Эпштейн Л. А. Методъ теории размерностей и подобия в задачах гидромеханики судов. Л.: Судостроение, 1970. 208 с.

47. Яворский Б. М., Пинский А. А. Основъ физики. Т. 1. М.: Физматлит, 2003. 576 с.

48. Appell P. Traité de mécanique rationnelle. Paris: Gauthier-Villars, 1893.

49. Appell P., Dautheville S. Précis de mécanique rationnelle. Paris: Gauthier-Villars, 1923.

50. Buckingham E. On physically similar systems. Illustrations of the use of dimensional equations // Phys. Rev., Ser. 2, 1914. vol.4, no.4. pp. 345-376. https://doi.org/10.1103/ physrev.4.345.

51. Ehrenfest-Afanassjewa T. A. Der Dimensionsbegriff und der analytische Bau physikalischer Gleichungen// Math. Ann., 1916. vol.77, no.2. pp. 259-276. https://doi.org/10.1007/ BF01456903.

52. Ehrenfest-Afanassjewa T. A. Dimensional analysis viwed from standpoint of the theore of similitude// Philos. Magazine, 1926. vol. 7, no. 1. pp. 257-272.

53. Reyleigh L. The principle of similitude// Nature, 1915. vol.95. pp. 66-68. https://doi. org/10.1038/095066c0.

54. Ruark A. E. Inspectional analysis: A method wich supplements dimensional analysis // J. Mitchell Soc., 1935. vol. 51. pp. 127-133.

55. Stokes G. On the effect of the internal friction of fluids on the motion of pendulums / Transactions of the Cambridge Philosophical Society. vol. IX, part II, 1850. pp. 8-106.

56. Tolman R. C. The principle of similitude// Phys. Rev., Ser. 2, 1914. vol. 3, no. 4. pp. 244255. https://doi.org/10.1103/physrev.3.244.

57. Tolman R. C. The principle of similitude and the principle of dimensional homogeneity // Phys. Rev., Ser. 2, 1915. vol.6. pp. 219-233.

58. Ehrenfest-Afanassjewa T. A. On Mr. R. C. Tolman's "Principle of Similitude" // Phys. Rev., Ser. 2, 1916. vol. 8. pp. 1-7. https://doi.org/10.1103/physrev.8.1. 


\title{
MSC: 00A73
}

\section{A review of methods for developing similarity criteria in mechanics}

\section{(C) N. V. Kramarenko}

Novosibirsk State Technical University,

20, pr. K. Marksa, Novosibirsk, 630073, Russian Federation.

\begin{abstract}
Similarity theory is the theoretical basis for modeling and drafting experiments. In addition, it can be used to conduct a comparative analysis of changes in the desired parameters of the problem without solving equations and without conducting experiments. All arguments in similarity theory are based on dimensionless power complexes, which are called similarity criteria (numbers), or invariants. In the literature of different years of release, various methods of obtaining similarity criteria are described, but the author was not able to find a unified classification of these methods and their comparison.

The article provides a review of various methods for obtaining similarity criteria, their classification, which includes five methods from differential equations and seven methods from dimension analysis. All methods are compared on a single problem of mechanics about forced vibrations of the load, which leads to four similarity numbers. This approach helps you compare the labor required to output similarity numbers in different ways. For each method, a list of references is given where it is mentioned, and a brief description of the tasks that are solved there. At the end is a summary table showing which methods are considered in the mentioned works. The table shows the relative popularity of methods.
\end{abstract}

Keywords: similarity theory, similarity methods, similarity criteria, similarity numbers, similarity indicators, dimensional analysis.

Received: $12^{\text {th }}$ June, 2020 / Revised: $2^{\text {nd }}$ March, 2021

Accepted: $10^{\text {th }}$ March, $2021 /$ First online: $29^{\text {th }}$ March, 2021

Competing interests. I declare that I have no apparent or potential conflicts of interest related to the publication of this article.

Authors' contributions and responsibilities. I take full responsibility for submitting the final manuscript in print. I approved the final version of the manuscript.

\section{Review Article}

( ) (1) The content is published under the terms of the Creative Commons Attribution 4.0 International License (http://creativecommons.org/licenses/by/4.0/)

Please cite this article in press as:

$\mathrm{Kr}$ a mar en ko N. V. A review of methods for developing similarity criteria in mechanics, Vestn. Samar. Gos. Tekhn. Univ., Ser. Fiz.-Mat. Nauki [J. Samara State Tech. Univ., Ser. Phys. Math. Sci.], 2021, vol. 25, no. 1, pp. 163-192. https://doi.org/10.14498/vsgtu1791 (In Russian).

Author's Details:

Nikolay V. Kramarenko (1) https://orcid.org/0000-0002-6301-1255

Cand. Techn. Sci., Associate Professor; Dept. of Aircraft Strength; e-mail: kramnv@rambler.ru; n.kramarenko@corp.nstu.ru 
Funding. This research received no specific grant from any funding agency in the public, commercial, or not-for-profit sectors.

\section{References}

1. Alabuzhev P. M., Geronimus V. V., Minkevich L. M., Shekhovtsov B. A. Teoriia podobiia i razmernostei. Modelirovanie [Theory of Similarity and Dimensionalities: Mathematical Modeling]. Moscow, Vyssh. shk., 1968, 206 pp. (In Russian)

2. Alabuzhev P. M., Kirnarskii M. Sh., Polishchuk V. G., Sokolov V. S., Chizhov A. E., Iushin V. V. Osnovy teorii podobiia, razmernosti, modelirovaniia [Basics of the Similarity Theory, Dimensionalities, and Modeling]. Kursk, Kursk Polytechnic Inst., 1993, 103 pp. (In Russian)

3. Arkhipov V. A., Berezikov A. P. Osnovy teorii inzhenerno-fizicheskogo eksperimenta [Basic Theory of Engineering Physics Experiment]. Tomsk, Tomsk Polytechnic Univ., 2008, 206 pp. (In Russian)

4. Barenblatt G. I. Scaling, self-similarity, and intermediate asymptotics. Dimensional Analysis and Intermediate Asymptotics, Cambridge Texts in Applied Mathematics, vol.14. Cambridge, Cambridge Univ. Press, 1996, xxii+386 pp. https://doi.org/10.1017/ CB09781107050242.

5. Barenblatt G. I. Dimensional Analysis. New York, Gordon \& Breach Sci. Publ., 1987, 135 pp.

6. Basniev K. S., Dmitriev N. M., Rozenberg G. D. Neftegazovaia gidromekhanika [Oil-Gas Hydromechanics]. Moscow, Izhevsk, Inst. Komp. Issled., 2005, 544 pp. (In Russian)

7. Birkhoff G. Hydrodynamics. A study in logic, fact and similitude. Princeton, New Jersey, Princeton Univ. Press, 1960, xi+184 pp.

8. Bridgman P. W. Dimensional Analysis. New Haven, Yale Univ. Press, 1932; Cambridge, Mass., Harvard Univ. Press, 1921.

9. Venikov V. A. Teoriia podobiia $i$ modelirovaniia (primenitel'no $k$ zadacham elektroenergetiki) [The Theory of Similarity and Modeling (Applied to the Problems of Electric Power Industry)]. Moscow, Vyssh. shk., 1976, 479 pp. (In Russian)

10. Vinnikov V. A., Karkashadze G. G. Gidromekhanika [Hydromechanics]. Moscow, Moscow State Mining Univ., 2003, 302 pp. (In Russian)

11. Gukhman A. A. Introduction to the Theory of Similarity. New York, Academic Press, 1965, $\mathrm{xxi}+256 \mathrm{pp}$.

12. D'iachkov Yu. A., Cheremshanov M. A. Modelirovanie sistem avtomobilestroeniia. Penza, Penza State Univ., 2009, 240 pp. (In Russian)

13. Kirpichev V. L. Besedy o mekhanike [Conversations about Mechanics]. Moscow, Leningrad, GITTL, 1950, 360 pp. (In Russian)

14. Kirpichev M. V. Teoriia podobiia [Theory of Similarity]. Moscow, USSR Acad. Sci., 1953, 96 pp. (In Russian)

15. Kirpichev M. V., Konakov P. K. Matematicheskie osnovy teorii podobiia [Mathematical Basics of the Similarity Theory]. Moscow, USSR Acad. Sci., 1949, 106 pp. (In Russian)

16. Kline S. J. Similitude and Approximation. New York, Springer Verlag, 1986, xix +229 pp. https://doi.org/10.1007/978-3-642-61638-9.

17. Kogan I. Sh. On the desirability of expanding the scope of application of the theory of similarity in teaching technical sciences, In: Mashinostroenie. Konstruktsii i tekhnologii [Mechanical Engineering. Designs and Technologies], vol. 3. Kirov, Vyatka State Technical Univ., 1998, pp. 64-69 (In Russian).

18. Korneev S. D. Gidrogazodinamika [Fluid and Gas Dynamics]. Moscow, Moscow State Industrial Univ., 2011, 230 pp. (In Russian)

19. Korpachev V. P. Teoreticheskie osnovy vodnogo transporta lesa [Theoretical Foundations of Waterborne Transport of Forests]. Moscow, Akad. Estestvoznaniia, 2009, 236 pp. (In Russian) 
20. Kramarenko N. V., Sitnov K. V. Similarity theory and special effects in cinema, In: Science. Industry. Defense (Novosibirsk, April 23-25, 2014). Novosibirsk, Novosibirsk State Technical Univ., 2014, pp. 347-351 (In Russian).

21. Kramarenko N. V., Kapustina A. A., Lavrina V. M., Skorobogatova A. A. Using similarity theory to conduct an engineering experiment, In: Science. Industry. Defense, vol. 1 (Novosibirsk, April 17-19, 2019). Novosibirsk, Novosibirsk State Technical Univ., 2019, pp. 96-101 (In Russian).

22. Kudinov A. A. Tekhnicheskaia gidromekhanika [Technical Hydromechanics]. Samara, Samara State Technical Univ., 2006, 295 pp. (In Russian)

23. Kutateladze S. S. Analiz podobiia $i$ fizicheskie modeli [Similarity Analysis and Physical Models]. Novosibirsk, Nauka, 1986, 296 pp. (In Russian)

24. Loitsianskii L. G. Mekhanika zhidkosti i gaza [Liquid and Gas Mechanics]. Moscow, Nauka, 1970, 904 pp. (In Russian)

25. Loitsianskii L. G. Mekhanika zhidkosti i gaza [Liquid and Gas Mechanics]. Moscow, Drofa, 2003, 840 pp. (In Russian)

26. Loitsianskii L. G. Similarity and dimensional methods in fluid and gas mechanics, In: Collection of Methodological Papers on Theoretical Mechanics, issue 11. Moscow, Vyssh. shk., 1981, 22-31 pp. (In Russian)

27. Martynov A. K. Prikladnaia aerodinamika [Applied Aerodynamics]. Moscow, Mashinostroenie, 1972, 448 pp. (In Russian)

28. Shvydkoi V. S. (ed.) Mekhanika zhidkosti i gaza [Liquid and Gas Mechanics]. Moscow, Akademkniga, 2003, 464 pp. (In Russian)

29. Morozov N. A. Osnovy kachestvennogo fiziko-matematicheskogo analiza i novye fizicheskie faktory, obnaruzhivaemye im v razlichnykh iavleniiakh prirody [Fundamentals of qualitative physical and mathematical analysis and new physical factors found by it in various natural phenomena]. Moscow, 1908, 402 pp. (In Russian)

30. Mkhitarian A. M. Aerodinamika [Aerodynamics]. Moscow, Mashinostroenie, 1976, 448 pp. (In Russian)

31. Some survey papers and primary sources on the history of pi-theorem and similarity theory (In Russian). Retrieved from http://gidropraktikum.narod.ru/pi-theorem-history. htm (March 29, 2021).

32. Osnovy teorii podobiia i modelirovaniia. Terminologiia. Sbornik rekomenduemykh terminov [Basics of the Similarity Theory and Modeling. Terminology. Compendium of Recommended Terms], issue 88. Moscow, Nauka, 1973, 50 pp. (In Russian)

33. Pinchuk S. I. Organizatsiia eksperimenta pri modelirovanii i optimizatsii tekhnicheskikh sistem [Organization of Experiment in Modeling and Optimization of Technical Systems]. Dnepropetrovsk, Diva, 2008, 248 pp. (In Russian)

34. Porodnov B. T. Chislennye metody resheniia zadach mekhaniki sploshnykh sred [Numerical Methods for Solving Problems of Continuum Mechanics]. Ekaterinburg, UGTU-UPI, 2008 (In Russian).

35. Romanov O. Ya., Khodosov V. V. Modelirovanie pri proektirovanii slozhnykh tekhnicheskikh sistem [Modeling in the Design of Complex Technical Systems]. St. Petersburg, Baltic State Technical Univ., 60 pp. (In Russian)

36. Sannikov R. Kh. Teoriia podobiia i modelirovaniia. Planirovanie inzhenernogo eksperimenta [Similarity and Modeling Theory. Planning of Engineering Experiment]. Ufa, Ufa State Petroleum Technical Univ., 2010, 253 pp. (In Russian)

37. Sedov L. I. Similarity and Dimensional Methods in Mechanics. New York, Academic Press, 1959, 363 pp. https://doi.org/10.1016/C2013-0-08173-X.

38. Sergel' O. S. Prikladnaia gidrogazodinamika [Applied Fluid and Gas Dynamics]. Moscow, Mashinostroenie, 1981, 374 pp. (In Russian)

39. Serrin J. Mathematical Principles of Classical Fluid Mechanics, Fluid Dynamics I. Berlin, Springer, 1959. https://doi.org/10.1007/978-3-642-45914-6_2. 
40. Silin S. S. Metod podobiia pri rezanii materialov [Similarity Methods in Metal Cutting]. Moscow, Mashinostroenie, 1979, 152 pp. (In Russian)

41. Sokolov V. A. Osnovy teorii podobiia $i$ analiza razmernostei $v$ neftegazodobyche [Fundamentals of the Theory of Similarity and Dimensional Analysis in Oil and Gas Production]. Ukhta, UGTU, 2001, 159 pp. (In Russian)

42. Federman A. On some general methods of integration of first-order partial differential equations, Proceedings of the Saint-Petersburg Polytechnic Institute. Section of Technics, Natural Science, and Mathematics, 1911, vol. 16, no. 1, pp. 97-155 (In Russian).

43. Huntley H. E. Dimensional Analysis, Dover Books on Intermediate and Advanced Mathematics. New York, Dover Publ., 1967.

44. Shapovalov L. A. Modelirovanie v zadachakh mekhaniki elementov konstruktsii [Modeling in the Problems of the Mechanics of Structural Elements]. Moscow, Mashinostroenie, 1990, 288 pp. (In Russian)

45. Schenck H. Theories of Engineering Experimentation. New York, McGraw-Hill Book Comp., 1961, x+239 pp.

46. Epshteyn L. A. Metody teorii razmernostei i podobiia v zadachakh gidromekhaniki sudov [Methods of the Dimensional Analysis and Similarity Theory in Problems of Ship Hydromechanics]. Leningrad, Sudostroenie, 1970, 208 pp. (In Russian)

47. Yavorsky B. M., Pinsky A. A. Fundamentals of Physics, vol.1. Moscow, Mir Publ., 1975, $544 \mathrm{pp}$.

48. Appell P. Traité de mécanique rationnelle. Paris, Gauthier-Villars, 1893.

49. Appell P., Dautheville S. Précis de mécanique rationnelle. Paris, Gauthier-Villars, 1923.

50. Buckingham E. On physically similar systems. Illustrations of the use of dimensional equations, Phys. Rev., Ser. 2, 1914, vol.4, no.4, pp. 345-376. https://doi.org/10.1103/ physrev.4.345.

51. Ehrenfest-Afanassjewa T. A. Der Dimensionsbegriff und der analytische Bau physikalischer Gleichungen, Math. Ann., 1916, vol. 77, no. 2, pp. 259-276. https://doi.org/10.1007/ BF01456903.

52. Ehrenfest-Afanassjewa T. A. Dimensional analysis viwed from standpoint of the theore of similitude, Philos. Magazine, 1926, vol. 7, no. 1, pp. 257-272.

53. Reyleigh L. The principle of similitude, Nature, 1915, vol.95, pp. 66-68. https://doi.org/ 10.1038/095066c0.

54. Ruark A. E. Inspectional analysis: A method wich supplements dimensional analysis, J. Mitchell Soc., 1935, vol. 51, pp. 127-133.

55. Stokes G. On the effect of the internal friction of fluids on the motion of pendulums, In: Transactions of the Cambridge Philosophical Society, vol. IX, part II, 1850, pp. 8-106.

56. Tolman R. C. The principle of similitude, Phys. Rev., Ser. 2, 1914, vol. 3, no. 4, pp. 244-255. https://doi.org/10.1103/physrev.3.244.

57. Tolman R. C. The principle of similitude and the principle of dimensional homogeneity, Phys. Rev., Ser. 2, 1915, vol. 6, pp. 219-233.

58. Ehrenfest-Afanassjewa T. A. On Mr. R. C. Tolman's "Principle of Similitude", Phys. Rev., Ser. 2, 1916, vol. 8, pp. 1-7. https://doi.org/10.1103/physrev.8.1. 